\title{
Spectral properties and origin of the radio halo in A3562
}

\author{
S. Giacintucci ${ }^{1,2,3}$, T. Venturi ${ }^{3}$, G. Brunetti ${ }^{3}$, S. Bardelli ${ }^{1}$, D. Dallacasa ${ }^{2,3}$, S. Ettori ${ }^{1}$, A. Finoguenov ${ }^{4}$, \\ A. P. Rao ${ }^{5}$, and E. Zucca ${ }^{1}$ \\ 1 INAF - Osservatorio Astronomico di Bologna, via Ranzani 1, 40127 Bologna, Italy \\ e-mail: tventuri@ira.cnr.it \\ 2 Dipartimento di Astronomia, Università di Bologna, via Ranzani 1, 40127 Bologna, Italy \\ 3 INAF - Istituto di Radioastronomia, via Gobetti 101, 40129 Bologna, Italy \\ ${ }^{4}$ Max-Planck-Institut für extraterrestrische Physik, 85740 Garching, Germany \\ 5 National Centre for Radio Astrophysics (TIFR), Pune University Campus, Post Bag No. 3, Ganeshkhind, Pune 411 007, India
}

Received 8 March 2005 / Accepted 20 May 2005

\begin{abstract}
We present a new detailed multiband study of the merging cluster A3562, in the core of the Shapley Concentration Supercluster. We analyzed new, low frequency radio data performed at $240 \mathrm{MHz}, 332 \mathrm{MHz}$ and $610 \mathrm{MHz}$ with the Giant Metrewave Radio Telescope (GMRT). The new GMRT data allowed us to carry out a detailed study of the radio halo at the centre of A3562, as well as of the head-tail radio galaxy J1333-3141 embedded in it, and of the extended emission around the peripheral cluster galaxy J1332-3146a. Thanks to the present observations we could derive the integrated spectrum of the radio halo with five data points in the frequency range $240 \mathrm{MHz}-1.4 \mathrm{GHz}$. Our data show a clear steepening of the total spectrum in this frequency range. Furthermore, by comparing the GMRT $332 \mathrm{MHz}$ image with a previously published VLA $1.4 \mathrm{GHz}$ image, we produced an image of the halo spectral index distribution. The image shows a very complex structure, with an average value of $\alpha_{332 \mathrm{MHz}}^{1.4 \mathrm{GHz}} \sim 1.5$ and a number of knots steepening up to $\sim 2$. We performed a combined morphological and statistical analysis using the radio images and the quantities derived from XMM-Newton and Chandra observations. We discuss our results in the light of particle re-acceleration processes in galaxy clusters. In particular, we outline an overall picture, consistent with the available radio and $\mathrm{X}$-ray data, in which the cluster merger kinematics, the injection of turbulence and $\mathrm{B}$-amplification induced by the merger between A 3562 and SC 1329-313 are jointly taken into account.
\end{abstract}

Key words. radio continuum: general - X-rays: galaxies: clusters - galaxies: clusters: individual: A3562

\section{Introduction}

Cluster radio halos belong to a particular class of steepspectrum $\left(\alpha>1, S \propto v^{-\alpha}\right)$ radio sources, which are identified with regions of diffuse emission on Mpc scale, with no obvious optical counterpart. They are located at the centre of X-ray luminous and massive galaxy clusters (for a recent review see Feretti 2003), and their number is steadily growing. They are produced by synchrotron radiation from a population of relativistic electrons, coexisting with the thermal electrons and protons in the intracluster medium (ICM). Their origin seems to be strictly connected to the X-ray properties of the hosting cluster and to the presence of cluster merger activity (Buote 2001; Schuecker et al. 2001), since radio halos have been found only in clusters with significant substructure both in the galaxy distribution and X-ray brightness.

Merging processes can generate turbulence, which may amplify the cluster magnetic fields and re-accelerate the relativistic electrons diffused in the ICM, producing the emission we observe from radio halos (see e.g. the review papers by Brunetti 2003, 2004; and Sarazin 2004). The connection between the relativistic plasma and the thermal ICM is supported by the data available thus far, which suggest the existence of a correlation between the halo radio power and the X-ray luminosity, temperature and mass of the hosting clusters (Colafrancesco et al. 1999; Liang et al. 2000; Govoni et al. 2001a; Bacchi et al. 2003). In particular, in a number of clusters with radio halo, Govoni et al. (2001b) showed that the extent and the shape of the halo emission closely follow the X-ray structure of the cluster on large scales. In particular higher radio brightness is associated with higher $\mathrm{X}$-ray brightness of the gas.

The radio halo studied in this paper is located in the galaxy cluster A3562, in the core region of the Shapley Concentration Supercluster, at a mean redshift of $z=0.05$. A3562 belongs to the A3558 complex, one of the most spectacular examples of cluster merger. The merging stage of this chain of three clusters (A3558, A3562 and A3556) and two groups of galaxies (SC 1327-312 and SC 1329-313) has been extensively outlined by studies in the optical and X-ray bands (Bardelli et al. 1996, 1998a,b, 2000, and 2002; Ettori et al. 1997, 2000), as well as in the radio band (Venturi et al. 1997, 1998, 1999, 2000 and 2003, hereinafter V00 and V03 respectively; Giacintucci et al. 2004, hereinafter G04). Recently, Finoguenov et al. (2004, 
hereinafter F04) carried out a detailed study of the hydrodynamic state of A3562 and the nearby companion SC 1329-313 through XMM-Newton observations. They showed that the region of A3562 and SC 1329-313 is clearly disturbed, suggesting a recent interaction between the two clusters. In particular F04 proposed that SC 1329-313 has recently ( 1 Gyr ago) passed North of A3562, inducing a sloshing of the cluster core in the North-South direction.

The A3562 halo is one of the smallest and the less powerful radio halos known to date, with a largest linear size of $\sim 470 \mathrm{kpc}$ and a total radio power at $1.4 \mathrm{GHz}$ of $P_{1.4 \mathrm{GHz}} \sim$ $1.1 \times 10^{23} \mathrm{~W} \mathrm{~Hz}^{-1}(\mathrm{~V} 03)^{1}$. The X-ray properties of $\mathrm{A} 3562$, such as the luminosity and temperature, are less extreme than those of the other clusters hosting a radio halo. In particular, $k T=5.1 \pm 0.2 \mathrm{keV}$, and $L_{\mathrm{X}, \mathrm{bol}}=2.2 \times 10^{44} \mathrm{erg} \mathrm{s}^{-1}$ (Ettori et al. 2000). For this reason the halo source in A3562 plays a special role in understanding the origin of radio halos and their connection to the X-ray properties of the intergalactic medium and the merging activity of the hosting cluster. As shown in V03, the A3562 halo follows and extends the correlations between the halo radio power and the cluster X-ray luminosity and temperature down to lower values of all quantities involved. This suggests that A3562-like halos may help us to understand the missing link between the massive and X-ray luminous clusters with a radio halo and those without (or undetected).

In this paper we present new low frequency radio data of A3562, obtained with the Giant Metrewave Radio Telescope (GMRT) at $240 \mathrm{MHz}, 332 \mathrm{MHz}$ and $610 \mathrm{MHz}$.

The observations are centered on the core of A3562 and cover also the region of the nearby group SC 1329-313, East of $\mathrm{A} 3562$, where a region of diffuse radio emission extends around the radio galaxy $\mathrm{J} 1332-3146 \mathrm{a}$, at the border of the $\mathrm{X}$-ray emission of the group (G04).

In Sects. 2 and 3 we present the new GMRT observations. In Sect. 4 we combine the new data with the $1.4 \mathrm{GHz}$ VLA data from V03, to discuss the morphological and spectral properties of the radio halo. The total radio spectrum of the halo is modelled in Sect. 5. In Sect. 6 we perform a combined radio/X-ray analysis of the A3562 region, using XMM-Newton (F04) and Chandra (Ettori et al. in preparation) observations. Finally, in Sect. 7 we propose a cluster merger scenario to account for the observed properties of A3562. Summary and conclusions are given in Sect. 8.

\section{GMRT low frequency radio observations}

We observed the region of A3562 and SC 1329-313 using the GMRT simultaneously at 240 and $610 \mathrm{MHz}$ on 15 January 2003, and at $332 \mathrm{MHz}$ on 19 February 2003, for a total of $\sim 7 \mathrm{~h}$ at each frequency.

The observations were carried out in spectral line mode with 64 channels at 240/610 MHz and 128 channels at $332 \mathrm{MHz}$, with a spectral resolution of $125 \mathrm{kHz}$.

The details of the observations are summarized in Table 1.

\footnotetext{
1 Throughout the paper we assume $H_{0}=70 \mathrm{~km} \mathrm{~s}^{-1} \mathrm{Mpc}^{-1}, \Omega_{\mathrm{m}}=$ 0.3 and $\Omega_{\Lambda}=0.7$. At the average redshift of the A3558 complex $(z=$ 0.05 ), this cosmology leads to a linear scale of $1 \operatorname{arcsec}=0.98 \mathrm{kpc}$.
}

The data calibration and reduction were performed using the NRAO Astronomical Image Processing System (AIPS) package. At 240/610 MHz the sources 3C 147 and 3C 286 were used to determine and correct for the bandpass shape and for the initial amplitude and phase calibration. At $332 \mathrm{MHz}$ only 3C 286 was used as primary calibrator. The secondary phase calibrators were 1248-199 and 1311-222 at 240/610 $\mathrm{MHz}$, 1311-222 at $332 \mathrm{MHz}$.

An accurate editing was needed to identify and remove the data affected by radio frequency interference (RFI), especially at $332 \mathrm{MHz}$ and $240 \mathrm{MHz}$. In order to find a compromise between the size of the data sets and the need to minimize bandwidth smearing within the primary beam, the central channels were averaged using the AIPS task SPLAT to 6 channels of $1 \mathrm{MHz}$ each at $240 \mathrm{MHz}$ and $2 \mathrm{MHz}$ each at $332 \mathrm{MHz}$. At $610 \mathrm{MHz}$ the central band was averaged to 1 single channel of $6 \mathrm{MHz}$.

After some steps of phase self-calibration, at each frequency we produced images in a wide range of resolutions using the wide-field imaging technique, with 25 facets covering a field of view in the range $1.4^{2} \div 3.5^{2} \mathrm{deg}^{2}$, depending on the frequency and resolution. The final images were combined with the task FLATN and corrected for the primary beam appropriate to the GMRT antennas. As a final step, since right ascension and declination of the final fields were referred to the observation epoch, we converted them to the $\mathrm{J} 2000$ epoch by means of the AIPS task REGRD.

The average noise achieved in the final full resolution and tapered images is $\sim 0.80 \mathrm{mJy} \mathrm{b}^{-1}$ at $240 \mathrm{MHz}, \sim 0.30 \mathrm{mJy} \mathrm{b}^{-1}$ at $332 \mathrm{MHz}$ and $\sim 0.15 \mathrm{mJy} \mathrm{b}^{-1}$ at $610 \mathrm{MHz}$. These values are a few times higher than the expected thermal noise. The residual amplitude calibration errors are of the order of $10 \%$ at $610 \mathrm{MHz}, 20 \%$ at $332 \mathrm{MHz}$, and $15 \%$ at $240 \mathrm{MHz}$.

\section{Radio images}

The GMRT is composed of 30 antennas: 14 are located in a $\sim 1.1 \mathrm{~km}$ central compact array and the remaining are distributed in a roughly $Y$ shaped configuration, with a maximum baseline of $\sim 25 \mathrm{~km}$. The resulting $u-v$ coverage of the GMRT ensures a good sensitivity both to compact and to extended sources, and allows deep imaging over a wide range of angular resolutions at all frequencies. Thus we produced images of A3562 at different resolutions, from few arcsec to tens of arcsec. The low resolution images allowed us to enhance the low surface brightness emission from the radio halo. Using the high resolution images, we could accurately determine the total flux density of the tail J1333-3141 and the point-like sources embedded in the halo emission. After subtraction of these sources, we could estimate the halo total flux density. The details of all the radio images presented in this paper are given in Table 2. The values of the flux density of the halo refer to the diffuse emission and are determined after the subtraction of the headtail J1333-3141 and of the point-like sources from the total flux density measured from the images. 
Table 1. Details of the GMRT observations for A3562 and SC 1329-313.

\begin{tabular}{|c|c|c|c|c|c|c|c|c|}
\hline $\begin{array}{c}\text { Frequency } \\
\mathrm{MHz}\end{array}$ & $\begin{array}{c}\text { Bandwidth } \\
\text { MHz }\end{array}$ & $\begin{array}{c}\mathrm{RA}_{J 2000} \\
\mathrm{~h}, \mathrm{~m}, \mathrm{~s}\end{array}$ & $\begin{array}{c}\operatorname{Dec}_{J 2000} \\
\circ,,^{\prime}, "\end{array}$ & Primary beam & $\begin{array}{c}F W H M, \text { PA } \\
\text { (full array) } \\
\quad,^{\circ}\end{array}$ & $\begin{array}{c}u-v \text { range } \\
\mathrm{k} \lambda\end{array}$ & $\begin{array}{c}\text { Obs. time } \\
\mathrm{h}\end{array}$ & $\begin{array}{c}\mathrm{rms} \\
\mathrm{mJy} \mathrm{b}^{-1}\end{array}$ \\
\hline 240 & 8 & 133330 & -314100 & 108 & $17.4 \times 11.5,11$ & $\sim 0.05-20$ & 7 & 0.80 \\
\hline 332 & 16 & 133330 & -314100 & 87 & $15.0 \times 8.0,26$ & $\sim 0.10-30$ & 7 & 0.30 \\
\hline 610 & 8 & 133330 & -314100 & 43 & $7.3 \times 5.00,7$ & $\sim 0.15-50$ & 7 & 0.15 \\
\hline
\end{tabular}

Table 2. Images details.

\begin{tabular}{cccccccc}
\hline \hline Source & $\begin{array}{c}\mathrm{RA}_{J 2000} \\
\mathrm{~h}, \mathrm{~m}, \mathrm{~s}\end{array}$ & $\begin{array}{c}\mathrm{Dec}_{J 2000} \\
{ }^{\prime},{ }^{\prime},\end{array}$ & $\begin{array}{c}v \\
\mathrm{MHz}\end{array}$ & $\begin{array}{c}F W H M, \mathrm{PA} \\
{ }^{\prime},{ }^{\circ}\end{array}$ & $\begin{array}{c}\mathrm{rms}^{2} \\
\mathrm{mJy} \mathrm{b}^{-1}\end{array}$ & $\begin{array}{c}S_{\text {tot }} \\
\mathrm{mJy}\end{array}$ & $\begin{array}{c}\text { LLS }^{1} \\
\mathrm{kpc}\end{array}$ \\
\hline J1333-3141 & 133331.6 & -314102 & 1400 & $41.9 \times 35.1,55$ & 0.05 & $109.3 \pm 10.9$ & $\sim 80^{2}$ \\
& 133331.6 & -314102 & 610 & $7.3 \times 5.0,7$ & 0.15 & $195.7 \pm 19.6$ & $\sim 80$ \\
& 133331.6 & -314102 & 332 & $15.0 \times 8.0,26$ & 0.30 & $293.3 \pm 58.7$ & $\sim 90$ \\
& 133331.6 & -314102 & 240 & $17.4 \times 11.5,11$ & 0.80 & $322.2 \pm 48.3$ & $\sim 90$ \\
Radio halo & 133332.0 & -314100 & 1400 & $41.9 \times 35.1,55$ & 0.05 & $20 \pm 2$ & $\sim 475$ \\
& 133332.0 & -314100 & 610 & $30.0 \times 20.0,7$ & 0.15 & $90 \pm 9$ & $\sim 470$ \\
& 133332.0 & -314100 & 332 & $41.9 \times 35.1,55$ & 0.25 & $195 \pm 39$ & $\sim 530$ \\
& 133332.0 & -314100 & 240 & $41.9 \times 35.1,55$ & 0.80 & $220 \pm 33$ & $\sim 350$ \\
\hline
\end{tabular}

${ }^{1}$ LLS $=$ Largest Linear Size.

${ }^{2}$ Value derived from the 15.0" $\times$ 8.0" ATCA image at $1.4 \mathrm{GHz}$ (V00 and Fig. 7 in this paper).
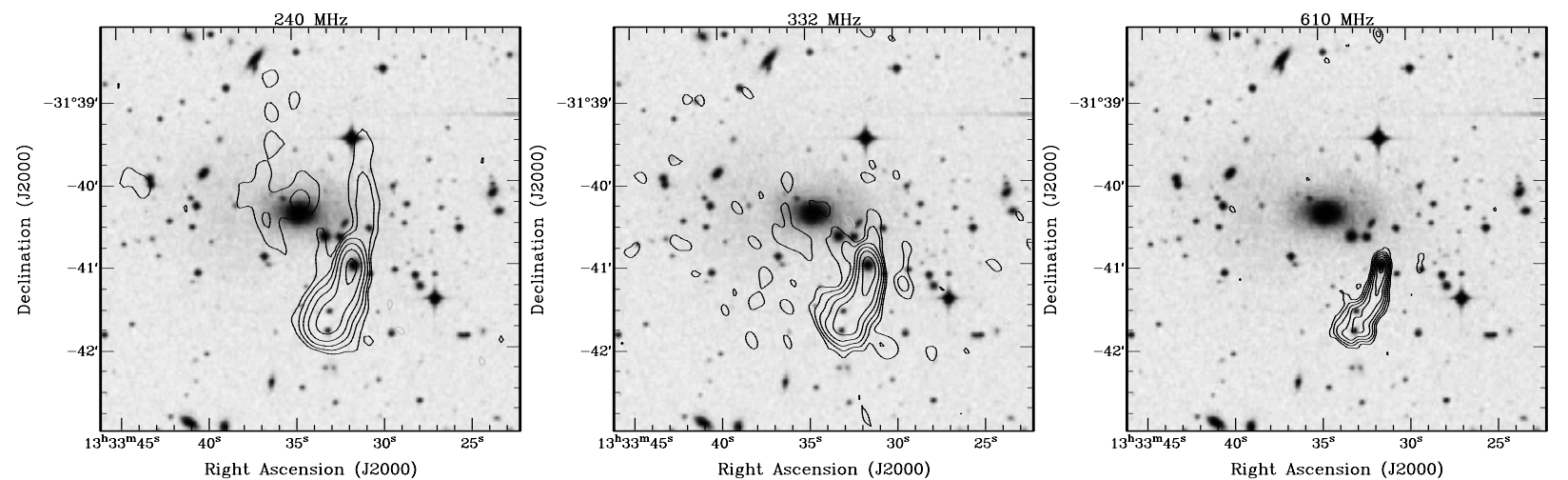

Fig. 1. Radio contours of the high resolution images of the head-tail radio galaxy J1331-3141 at $240 \mathrm{MHz}$ (left), $332 \mathrm{MHz}$ (centre) and $610 \mathrm{MHz}$ (right), overlaid on the DSS-1 optical frame. The rms noise is $0.80,0.30$ and $0.15 \mathrm{mJy} \mathrm{b}^{-1}$ respectively. Contour levels are $-2.4,2.4$, $4.8,9.6,19.2,38.4,76.8 \mathrm{mJy} \mathrm{b}^{-1}$ at $240 \mathrm{MHz},-0.9,0.9,1.8,3.6,7.2,14.4,28.8,57.6 \mathrm{mJy} \mathrm{b}^{-1}$ at $332 \mathrm{MHz},-0.45,0.45,0.9,1.8,3.6,7.2,14.4$, $28.8 \mathrm{mJy} \mathrm{b}^{-1}$ at $610 \mathrm{MHz}$. The angular resolution is $17.4^{\prime \prime} \times 11.5^{\prime \prime}$ in PA $11^{\circ}$ at $240 \mathrm{MHz}, 15.0^{\prime \prime} \times 8.0^{\prime \prime}$ in PA $26^{\circ}$ at $332 \mathrm{MHz}$ and $7.3^{\prime \prime} \times 5.0^{\prime \prime}$ in $\mathrm{PA} 7^{\circ}$ at $610 \mathrm{MHz}$.

\subsection{High resolution images}

In Fig. 1 we present the high resolution images of the centre of A3562, where the head-tail radio galaxy J1333-3141 is clearly visible. Despite the high resolution, residuals of the surrounding diffuse emission from the radio halo are visible at $240 \mathrm{MHz}$ and $332 \mathrm{MHz}$.

J1333-3141 is associated with the cluster elliptical galaxy MT $4108\left(b_{J}=17.25, v=14438 \mathrm{~km} \mathrm{~s}^{-1}\right)$, located at a projected distance of $\sim 1^{\prime}$ from the cluster dominant $\mathrm{cD}$ galaxy (V00). A detailed study of this source was carried out by V03, using VLA data at $330 \mathrm{MHz}, 1.4,4.86$ and $8.46 \mathrm{GHz}$, in addition to older ATCA data at $1.38 \mathrm{GHz}$ and $2.36 \mathrm{GHz}$ published in V00.

\subsection{Low resolution images}

At each frequency, the low resolution images of the radio emission from A3562 were produced using the full array with a taper, in order to weight down long baseline data points, preserving the total number of visibility points. Given the $u-v$ range at each frequency (see Table 1), the best compromise between the low resolution needed and the sensitivity requested to reliably image and analyse the radio halo was reached tapering the data to resolutions in the range $30^{\prime \prime}-40^{\prime \prime}$. For a proper comparison with the VLA $1.4 \mathrm{GHz}$ image of the halo in V03, we present here $240 \mathrm{MHz}$ and $332 \mathrm{MHz}$ images made with the same resolution, i.e. $41.9^{\prime \prime} \times 35.1^{\prime \prime}$, in PA $55^{\circ}$. Since we did not use the $610 \mathrm{MHz}$ data for a point-to-point analysis with the 


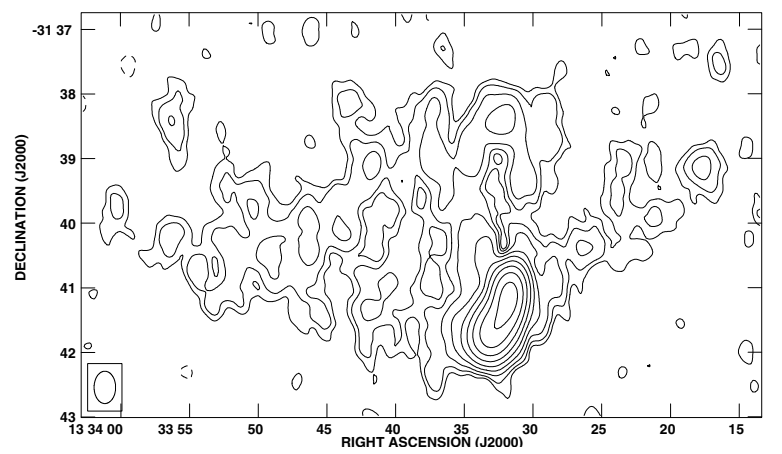

Fig. 2. Low resolution image at $610 \mathrm{MHz}$ of the central radio halo in A 3562. The restoring beam is $30^{\prime \prime} \times 20^{\prime \prime}$. Contour levels are $-0.5,0.25$, $0.5,1.0,2.0,4.0,8.0,16.0,32.0,64.0 \mathrm{mJy} \mathrm{b}^{-1}$.

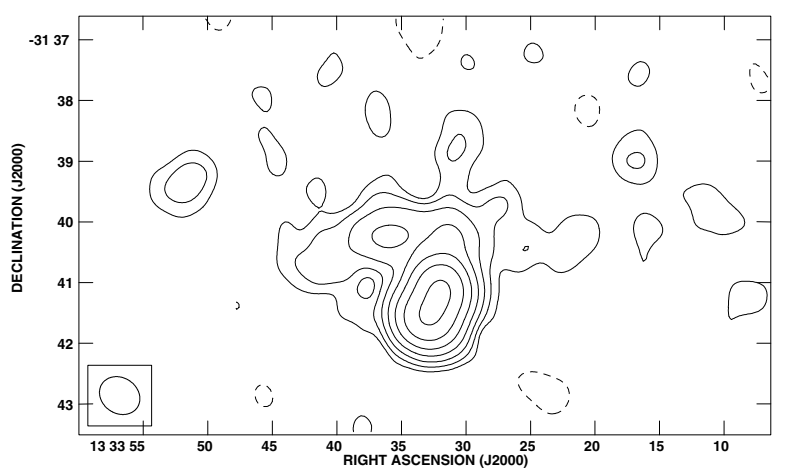

Fig. 3. Low resolution image at $240 \mathrm{MHz}$ of the central radio halo in A3562. The restoring beam is $41.9^{\prime \prime} \times 35.1^{\prime \prime}$. Contour levels are -2.4 , 2.4, 4.8, 9.6, 19.2, 38.4, 76.8, 153.6, 307.2, $614.4 \mathrm{mJy} \mathrm{b}^{-1}$.

VLA $1.4 \mathrm{GHz}$ data, in Fig. 2 we present a $610 \mathrm{MHz}$ image of the radio halo with the resolution of $30^{\prime \prime} \times 20^{\prime \prime}$, which better highligths the small scale features of the source.

Figure 3 shows the $240 \mathrm{MHz}$ contours of the radio halo, and Fig. 4 shows the radio emission at $332 \mathrm{MHz}$ from the whole region of A3562 and SC 1329-313, overlaid on the DSS-1 optical image, including both the radio halo and the diffuse source J1332-3146a in the SC group.

As clear from Figs. 2-4, the overall morphology of the radio halo at all frequencies shows the same irregular and complex structure, which extends in the NE-SW direction. This is similar to what is found at $1.4 \mathrm{GHz}$ (V03 and Fig. 9 in this paper). The largest angular size (LLS) is $\sim 8^{\prime}(\sim 475 \mathrm{kpc})$ at $1.4 \mathrm{GHz}$ and $610 \mathrm{MHz}$ and $\sim 9^{\prime}(\sim 530 \mathrm{kpc})$ in the $332 \mathrm{MHz}$ image (see Table 2). Note that these values do not include the extension of the wester filament (see Sect. 3.3).

The noise in the $240 \mathrm{MHz}$ image (Fig. 3) is considerably higher than in the other images $\left(0.80 \mathrm{mJy} \mathrm{b}^{-1}\right)$, therefore the lowest contour is ten times higher than at $610 \mathrm{MHz}$ (Fig. 2), and the total size of the halo at $240 \mathrm{MHz}$ is smaller than at the other frequencies. However, there is a clear indication that the region covered by the halo emission is consistent with the morphology in Figs. 2 and 4. For this reason, in order to determine the total flux density of the radio halo at $240 \mathrm{MHz}$, we integrated the measurement over the whole region of emission covered at the other three frequencies (see Table 2). This value is considerably higher than the one derived integrating only the portion of emission included in the $3 \sigma$ level (from RA $=13^{\mathrm{h}} 33^{\mathrm{m}} 20^{\mathrm{s}}$ to $13^{\mathrm{h}} 33^{\mathrm{m}} 45^{\mathrm{s}}$ ), in support of the presence of positive residuals in this region. In particular, the flux density within the $3 \sigma$ level is $S_{\text {tot }} \sim 170 \mathrm{mJy}$, i.e. $60 \mathrm{mJy}$ less than the value given in Table 2 . For comparison, the flux density measured in regions of similar size outside the cluster centre, is of the order of the image noise. As a further check of our estimate of the radio halo size at $240 \mathrm{MHz}$, we integrated the flux density over an even wider region, obtaining flux density values consistent with the one given in Table 2 (within the errors).

The linear size of this radio halo is among the smallest found thus far. This is not unexpected, since giant radio halos (LLS > $1 \mathrm{Mpc}$ ) are usually found in massive and X-ray luminous galaxy clusters, while A3562 does not show extreme $\mathrm{X}$-ray properties (see Sect. 1).

The diffuse source J1332-3146a is visible in the bottomright part of Fig. 4. This source is associated with the brightest galaxy in SC 1329-313. Its extended emission at $332 \mathrm{MHz}$ is strongly elongated to the North-East direction and points toward the centre of A3562. The full resolution images at $240 \mathrm{MHz}$ and $332 \mathrm{MHz}$ of the region of the J1332-3146a source (not presented here) show no sign of the compact component associated with the optical galaxy nucleus and detected at $610 \mathrm{MHz}$ (image not shown here) and at higher frequency (V00, G04). This suggests that the radio nucleus of the optical counterpart is self-absorbed at low frequencies, and that the extended radio emission is not connected to the present activity of the AGN. We also note that the radio spectrum of J1332-3146a is steep, with a spectral index between $240 \mathrm{MHz}$ and $1.4 \mathrm{GHz}$ (G04) $\alpha_{240 \mathrm{MHz}}^{1.4 \mathrm{GHz}}=1.2 \pm 0.1$.

\subsection{General comments on the radio emission in A3562}

Our low resolution images show that the radio emission in A3562 is characterised by some interesting and peculiar features, and suggest some connection between the centre of A3562 and the region of SC 1329-313. The radio halo has an irregular shape, with a filament of emission pointing South-West, toward SC 1329-313. This is particularly clear at $332 \mathrm{MHz}$ (Fig. 4) and at $1.4 \mathrm{GHz}$ (V03, and also Fig. 9 in this paper). The $332 \mathrm{MHz}$ image in Fig. 4 shows also that J13323146a is extended in the direction of A3562, and that positive residuals (at the $3 \sigma$ level, and higher) are present between these two regions. These features were noted also at $1.4 \mathrm{GHz}(\mathrm{G} 04)$, and the combination of these two pieces of information is suggestive of the possibility that very low brightness emission, undetectable with the present radio instruments, permeates the region between the centre of A3562 and SC 1329-313.

\section{Spectral index images and integrated spectra}

Spectral index imaging and integrated radio spectra are powerful tools in our understanding of the origin of the radio halo emission and of the re-acceleration processes on cluster scales. 


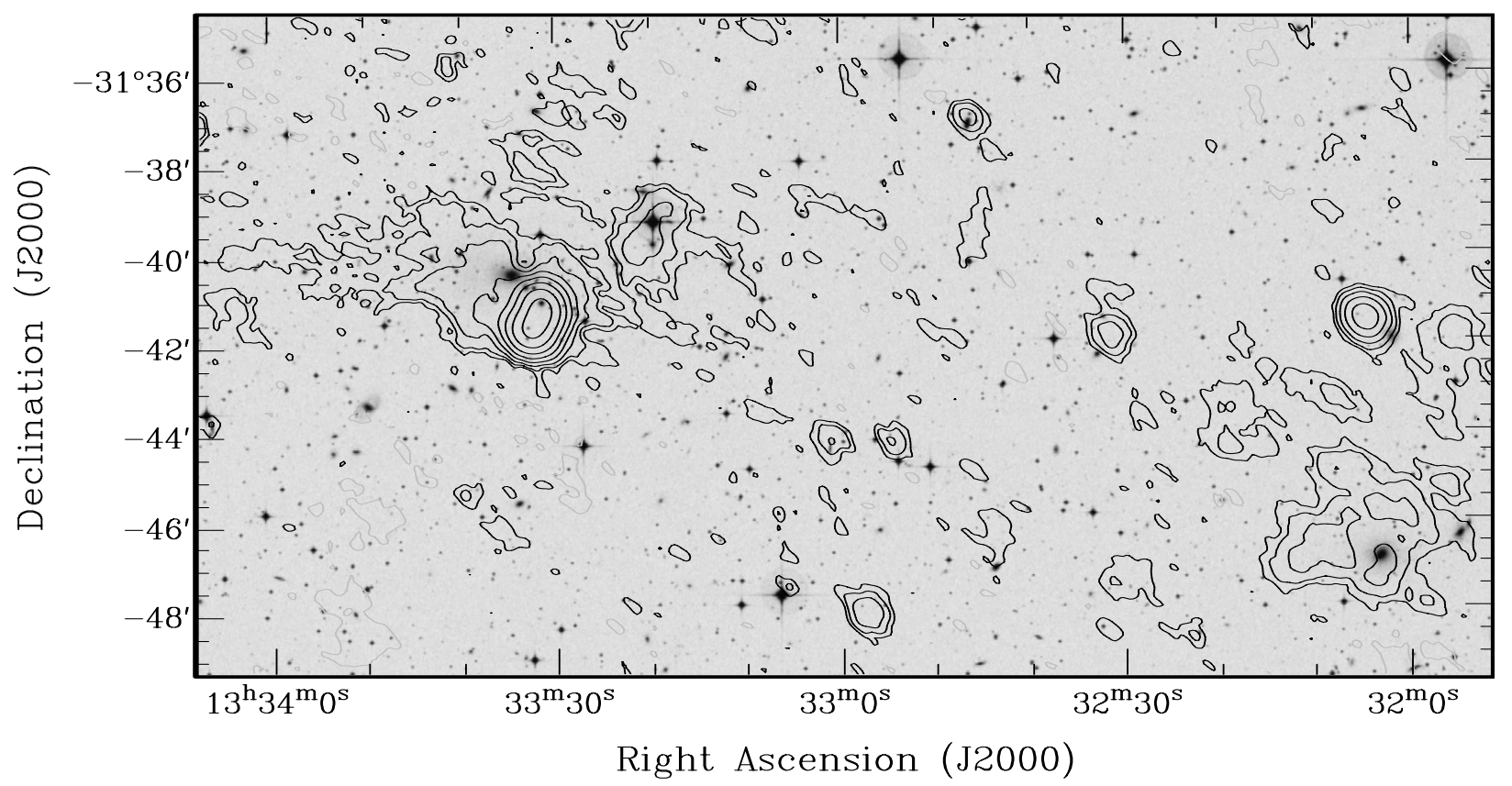

Fig. 4. Low resolution image at $332 \mathrm{MHz}$ of the region including the centre of A3562 and the extended radio galaxy J1332-3146a (on the right) overlaid on the optical DSS-1 frame. The restoring beam is $41.9^{\prime \prime} \times 35.1^{\prime \prime}$. Contour levels are $-1,1,2,4,8,16,32,64,128 \mathrm{mJy} \mathrm{b}^{-1}$.

However, only very recently it has become possible to make spectral index maps of radio halos, and to fill the low frequency domain $(v<1.4 \mathrm{GHz})$ in their integrated spectra. In particular, at present spectral index maps are available only for a couple of cluster radio halos (Feretti et al. 2004, and ref. therein), while a good total spectrum is available only for Coma-C, at the centre of the Coma cluster (Thierbach et al. 2003), and for the central region of the radio halo in the cluster 1E 0657-56 (Liang et al. 2000).

Using the data presented in this paper and those published in V00, V03 and G04, we produced the images of the spectral index distribution for the radio halo (Sect. 4.1) and the headtail radio galaxy J1333-3141 (Sect. 4.2). Moreover, we derived the total radio spectrum down to $240 \mathrm{MHz}$ for both sources.

\subsection{The radio halo}

In order to determine the distribution of the spectral index over the radio halo, we compared the GMRT image at $332 \mathrm{MHz}$ (Fig. 4) and the VLA image at $1.4 \mathrm{GHz}$ (Fig. 9), produced with the same cell size and restoring beam $\left(F W H M=41.9^{\prime \prime} \times\right.$ $35.1^{\prime \prime}$, in PA $\left.55^{\circ}\right)$. The images were aligned using the AIPS task HGEOM, clipped at a $3 \sigma$ level and combined to create the spectral index map within the Synage++ package (Murgia 2001). We note that the shortest baseline is the same at $332 \mathrm{MHz}$ (Table 1) and $1.4 \mathrm{GHz}$ (V03).

Figure 5 shows the spectral index image of the halo (colours) with $332 \mathrm{MHz}$ contours overlaid. It is clear that the region of J1333-3141 has a flat to normal spectrum, with $\alpha_{332 \mathrm{MHz}}^{1.4 \mathrm{GHz}}$ in the range $\sim 0 \div 0.7$. The spectrum of the radio halo is steep, with an average value $\alpha_{332 \mathrm{MHz}}^{1.4 \mathrm{GHz}} \simeq 1.5 \pm 0.1$ and knots steepening up to $\alpha_{332 \mathrm{MHz}}^{1.4 \mathrm{GHz}} \simeq 2.0 \pm 0.2$. Inspection of Fig. 5 suggests that there is a clear separation between the region
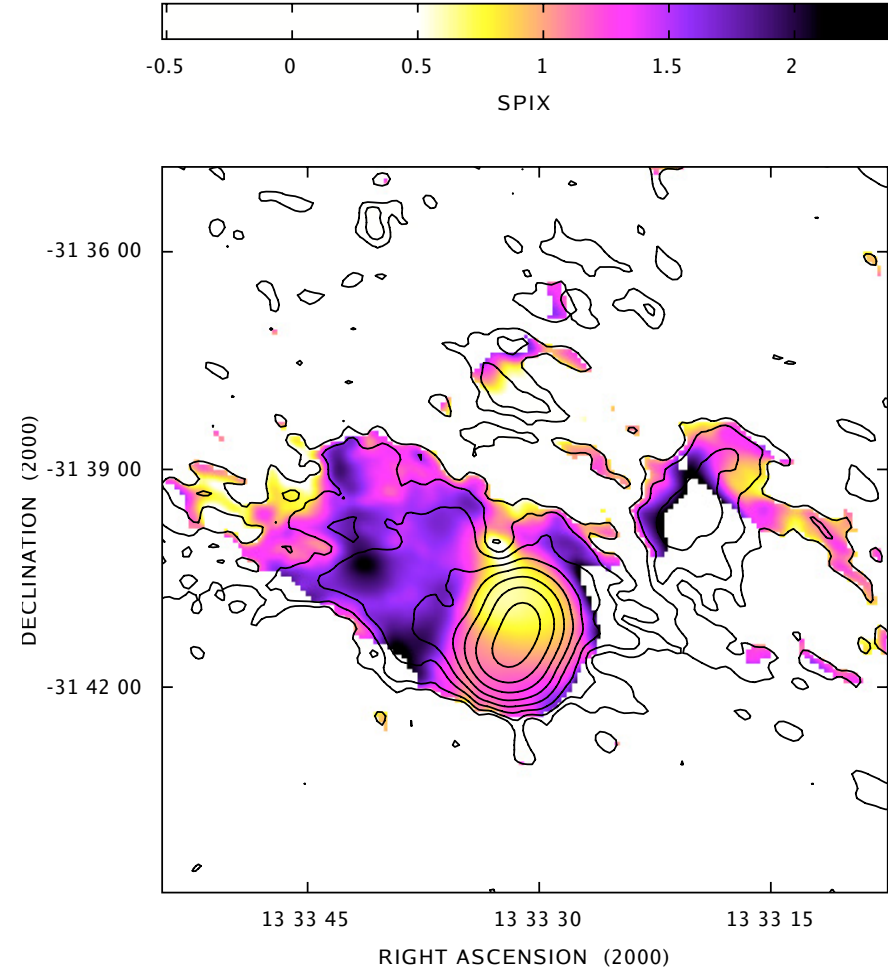

Fig. 5. Color scale image of the spectral index distribution over the radio halo between $332 \mathrm{MHz}$ and $1400 \mathrm{MHz}$, as computed from images with a restoring beam of $41.9^{\prime \prime} \times 35.1^{\prime \prime}$, in PA $55^{\circ}$. Overlaid are the GMRT $332 \mathrm{MHz}$ radio contours at levels $-1,1,2,4,8,16,32,64$, $128 \mathrm{mJy} \mathrm{b}^{-1}$.

dominated by the head-tail and the region dominated by the halo, i.e. there is a sharp transition in the spectral index distribution of these two components, being the latter substantially steeper. 


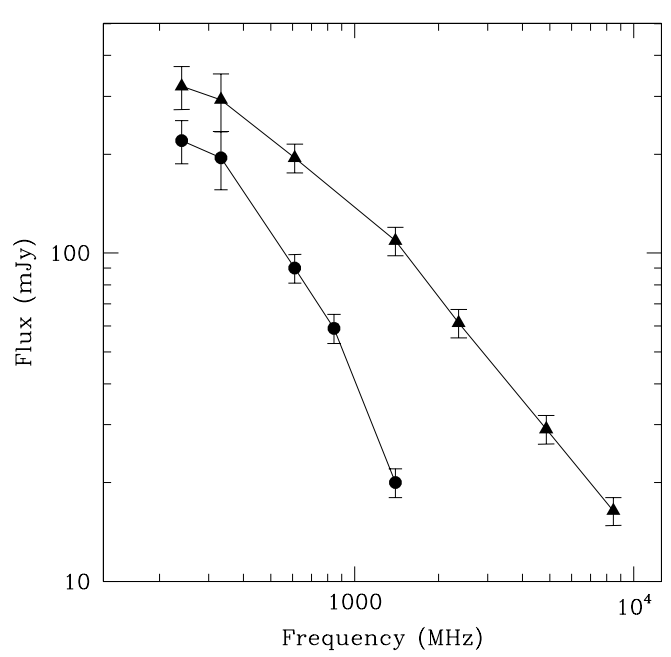

Fig. 6. Radio spectra of the halo source (filled dots) and of the headtail J1333-3141 (triangles) in A3562. The $1.4 \mathrm{GHz}$ flux density values are taken from V03. The $843 \mathrm{MHz}$ value for the halo is from V00. For the head-tail, the $2.36 \mathrm{GHz}$ flux density is taken from V00, and the $330 \mathrm{MHz}, 4.86 \mathrm{GHz}$ and $8.46 \mathrm{GHz}$ values are from $\mathrm{V} 03$.

We determined the integrated synchrotron spectrum of the radio halo in the frequency range $240 \mathrm{MHz}-1400 \mathrm{MHz}$, shown in Fig. 6 (filled dots; see also Sect. 5.1 for discussion), using the values of the flux density reported in Table 2 and the MOST $843 \mathrm{MHz}$ image in V00, who reported a flux density of $S_{843 \mathrm{MHz}}=59 \pm 6 \mathrm{mJy}$ for the halo emission. The radio spectrum of the halo appears to be very steep between $1400 \mathrm{MHz}$ and $843 \mathrm{MHz}$, with a spectral index $\alpha_{843 \mathrm{MHz}}^{1400 \mathrm{MHz}}$ in the range 1.9-2.3 (V03). Between $843 \mathrm{MHz}$ and $332 \mathrm{MHz}$ the spectral index is $\alpha_{332 \mathrm{MHz}}^{843 \mathrm{MHz}} \sim 1.3 \pm 0.2$, and below $332 \mathrm{MHz}$ the spectrum flattens to $\alpha_{240}^{332} \sim 0.4 \pm 0.7$.

\subsection{The head-tail radio galaxy $\mathrm{J} 1333-3141$}

In Fig. 7 we show the spectral index image of J1333-3141 (colours), obtained by comparison of the GMRT $332 \mathrm{MHz}$ image and the ATCA map at $1.38 \mathrm{GHz}$ (contours from V00), produced with the same cell size and restoring beam $(F W H M=$ $15.0^{\prime \prime} \times 8.0^{\prime \prime}$, in PA $\left.26^{\circ}\right)$. As described for the radio halo in Sect. 4.1, the maps were aligned and clipped at a $3 \sigma$ level before combining them.

The spectral index distribution clearly shows that we are dealing with a tailed radio galaxy. The flattest region $\left(\alpha_{1.38 \mathrm{GHz}}^{332 \mathrm{MHz}} \sim 0\right)$ is coincident with the core region imaged at high resolution and high frequency in V03, and the significant transverse steepening corresponds to the tail beginning (the peak in the $1.38 \mathrm{GHz}$ image). The spectral index smoothly steepens along the tail up to a value of $\sim 1$.

The total synchrotron spectrum of the source J1333-3141 in the frequency range $240 \mathrm{MHz}-8.46 \mathrm{GHz}$ is shown in Fig. 6 (triangles). The spectrum can be described as a power law with $\alpha_{610 \mathrm{MHz}}^{8.46 \mathrm{GHz}}=0.96 \pm 0.04$, with a flattening at frequencies $v<332 \mathrm{MHz}$. Figure 6 also highlights the well known different spectral shape of halo sources and extended radio galaxies at frequencies $v>330 \mathrm{MHz}$ (e.g. the halo source Coma-C,

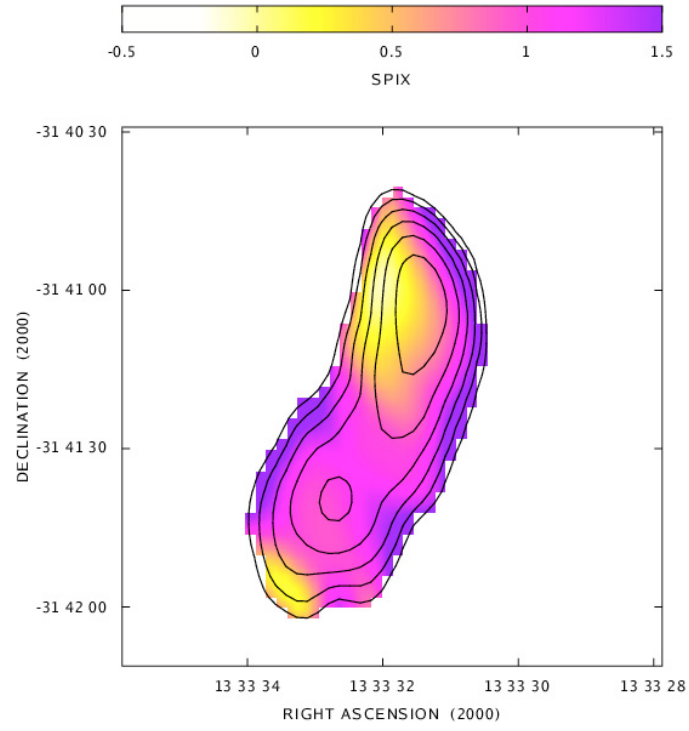

Fig. 7. Color scale image of the spectral index distribution over the head-tail radio galaxy J1333-3141, between $332 \mathrm{MHz}$ and $1380 \mathrm{MHz}$, as computed from images with a restoring beam of $15.0^{\prime \prime} \times 8.0^{\prime \prime}$, in PA $26^{\circ}$. Overlaid are the ATCA $1380 \mathrm{MHz}$ radio contours at levels $-0.5,0.5,1.0,2.0,4.0,8.0,16.0 \mathrm{mJy} \mathrm{b}^{-1}$.

Thierbach et al. 2003, and low luminosity radio galaxies, Parma et al. 2002).

\section{Origin of the radio halo}

The origin of the diffuse radio emission found in galaxy clusters is still an open issue. A promising possibility for the explanation of radio halos is provided by electron acceleration due to merger driven turbulence (e.g. Schlickeiser et al. 1987; Brunetti et al. 2001a,b; Petrosian 2001).

During cluster mergers, the infall of the minor subcluster through the main one generates turbulent velocity fields in the ICM. This has been observed in the hydrodynamical simulations of merging galaxy clusters, in the case of both head-on and off-axis collisions (Roettiger et al. 1997; Ricker \& Sarazin 2001). Turbulence might be powered by the developing of large scale instabilities at the head of the colliding subcluster and by the dark matter oscillations which take place during a cluster merger. The large scale turbulent eddies diffuse through the cluster volume and decay into smaller scale turbulence which may accelerate relativistic particles. A fraction of the energy flux of the fluid turbulence is likely channelled into magnetohydrodynamical (MHD) waves, e.g. fast magnetosonic (MS) waves and Alfvén waves (e.g. Eilek 1979; Fujita et al. 2003; Brunetti et al. 2004).

Unfortunately the limited dynamic range of the present numerical simulations does not allow a detailed investigation of the connection between cluster mergers and particle acceleration, in particular the cascading of the turbulent eddies toward the smaller scales and the generation of MHD modes. On the other hand a simplified Montecarlo approach to the problem allows a statistical investigation on the connection between mergers and particle acceleration: a first attempt in this direction has been recently developed by Cassano \& Brunetti (2005). 
Following those calculations, mergers between subclumps with mass ratio in the range 2-5 are the responsible for the injection of the bulk of fluid turbulence in galaxy clusters and are expected to be those which might activate giant radio halos in the most massive clusters. Although these calculations are based on the simplified assumption that mergers are essentially headon collisions, the main results should not be sensitive to the details of the impact parameter, provided that the subclump goes across the innermost regions of the massive cluster (i.e. within $0.2-0.3$ virial radii).

The spectrum of the electrons accelerated by turbulence has a maximum energy which is due to the balance between radiative losses and acceleration terms. The main feature of the synchrotron spectrum emitted by these electrons is the steepening at high radio frequencies, provided that the maximum accelerated energy is of the order of that of the radio emitting electrons.

\subsection{Study of the integrated radio spectrum}

In Fig. 8 we report the spectrum of the radio halo in A3562 (see Sect. 4.1) compared to the expectations from different models for the origin of the radio emitting electrons (see figure caption). Note that the spectrum is given as $v-v F(v)$.

Despite the relatively large error bars at lower frequencies, the high number of data points and their statistical trend strongly constrain the current models for the formation of this radio halo. It is clear that the observations are not consistent with a simple power law spectrum (e.g., as expected from secondary models; Dennison 1980; Blasi \& Colafrancesco 1999). The A3562 halo is among the few ones which show a steepening of the radio spectrum with frequency: this provides evidence for diffuse particle acceleration in the emitting region. In the specific particle turbulent-acceleration model, which seems to better describe the observed spectral behaviour (solid line), we assume that the electrons are accelerated and emit in a region where the magnetic field strength is approximately constant (reasonably assumed of the order of $\mu \mathrm{G}$ ). This implies that the scale of the diffuse radio source is comparable to (or smaller than) the scale of the variation of the field intensity in the ICM. The magnetic field in the ICM is believed to be amplified during the cluster formation mostly due to dynamo amplification and shear flows and thus it should decrease with distance from the cluster centre. Detailed MHD numerical simulations show that the radial profile of the field intensity in massive clusters is expected to be almost flat in the core region, and it rapidly decreases with distance (Dolag et al. 2002). The average size of the halo in A 3562 is of the order of the core radius and this is consistent with the above scenario in which particles are emitting in a region with relatively uniform field intensity.

\section{X-radio analysis}

In order to complete our analysis on the A3562 radio halo and to understand its origin and connection with the cluster merger history, we performed a composite X-radio study by using (a) the radio image at $1.4 \mathrm{GHz}$ first presented in V03;

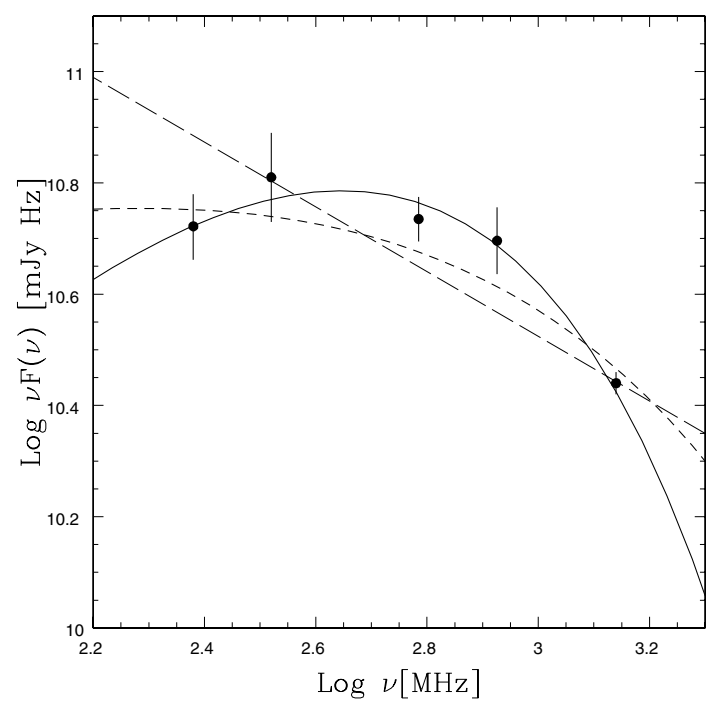

Fig. 8. Energy spectrum of the radio halo in A3562 compared with the expectations from three models for the origin of the emitting electrons. The long dashed line represents the emission from a power law energy distribution of the emitting electrons; this model is normalized at the $332-1400 \mathrm{MHz}$ emission. The short dashed line is a turbulentacceleration model of electrons which are accelerated and emit in a region which is larger than the typical scale of variation of the magnetic field intensity (the slope of the model is similar to what is reported in Brunetti et al. 2004, Fig. 21). The solid line is obtained assuming that particles are accelerated with a fixed acceleration efficiency and emit in a region of constant magnetic field intensity.

(b) XMM-Newton data first presented in F04; (c) Chandra observations carried out in ACIS-I with an exposure of $\sim 20 \mathrm{ks}$ (Ettori et al. in preparation).

\subsection{X-radio morphological comparison}

Figure 9 shows the overlay of the radio contours at $1400 \mathrm{MHz}$ (V03) on the XMM-Newton mosaic of the A3562 region, obtained by F04 in the $0.8-2 \mathrm{keV}$ band.

In Figs. 10 and 11 we compare the radio emission of the halo at $1400 \mathrm{MHz}$ with the pseudo-entropy and pseudopressure maps of A3562, derived by F04 and defined as $S=$ $T / \sqrt[3]{I}$ and and $P=T \times \sqrt{I}$ respectively (Churazov et al. 2003; Briel et al. 2003), where $I$ is the surface brightness in the 0.8-2 $\mathrm{keV}$ band and $T$ is the temperature. Figure 9 clearly indicates that A3562 and SC 1329-313 "feel each other" both in the radio and X-ray bands. In particular:

i) the X-ray brightness of A3562 is slightly elongated toward SC 1329-313, whose emission strongly points to the centre of A3562;

ii) the weak filament of radio emission at the western end of the halo points toward SC 1329-313, while the extended emission of J1332-3146a (located in SC 1329-313) is strongly elongated toward A3562;

iii) the radio halo extends mostly along the North-East direction.

We point out that the radio and X-ray emission in A3562, the radio galaxy $\mathrm{J} 1332-3146 \mathrm{a}$ and the X-ray emission of 


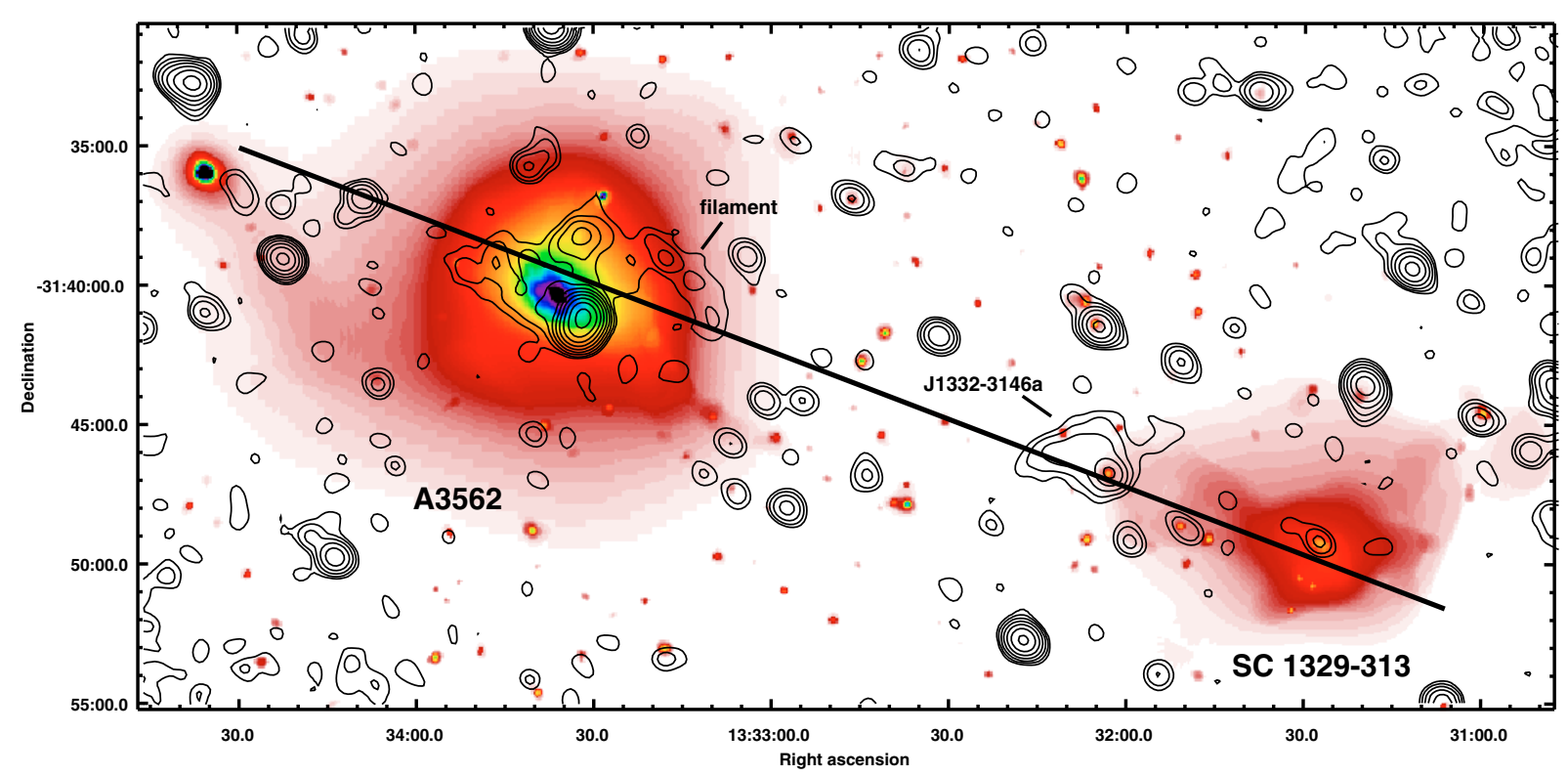

Fig. 9. VLA-1400 MHz contours of the radio emission from A3562 and SC 1329-313 superimposed on the XMM-Newton mosaic in the $0.8-2 \mathrm{keV}$ band. The radio levels are $-0.15,0.15,0.3,0.6,1.2,2.4,4.8,9.6,19.2,38.4,76.8$ and $153.6 \mathrm{mJy} \mathrm{b}^{-1}$. The restoring beam is $F W H M=41.9^{\prime \prime} \times 35.1^{\prime \prime}$, in p.a. $55^{\circ}$.

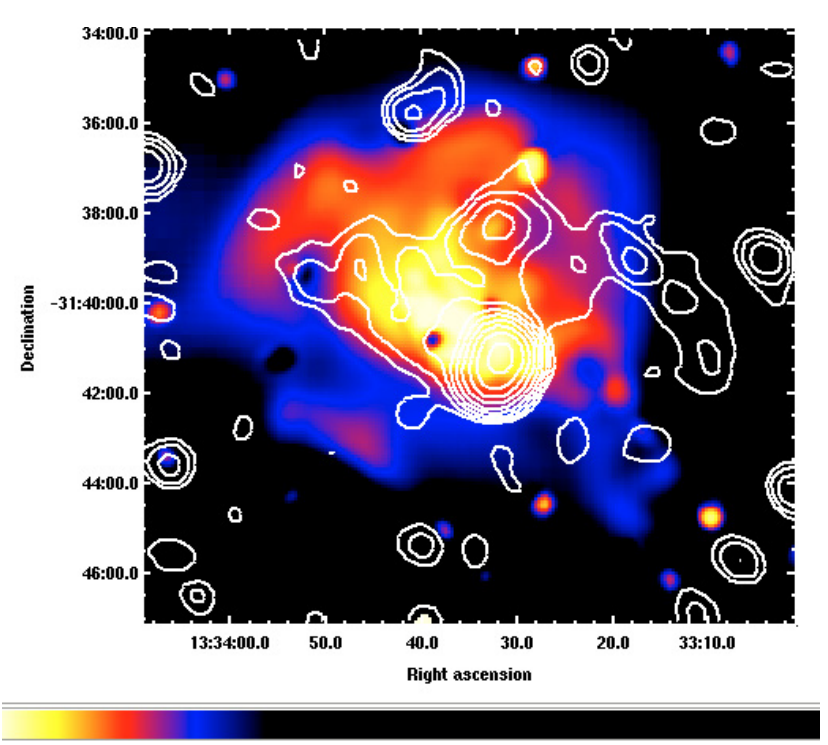

Fig. 10. VLA-1400 MHz image of the radio halo at the centre of A3562, overlaid on the XMM-Newton pseudo-entropy map of the cluster. Radio levels are $0.00015 \times(-1,1,2,4,8,16,32,64,128,256$, $512,1024) \mathrm{Jy} \mathrm{b}^{-1}$ and the resolution is $41.9^{\prime \prime} \times 35.1^{\prime \prime}$.

SC 1329-313 are almost perfectly aligned, as indicated from the line in Fig. 9.

Figures 10 and 11 zoom into the A3562 centre. It is noteworthy that the pressure, the entropy and the halo radio emission extend to North-East with respect to the cluster centre. The inner region of the pseudo-entropy distribution shows two tails toward North. Hereinafter, we will refer to these features as to the pseudo-entropy tails. The South-Eastern part of the radio halo has a sharp edge, and closely follows the morphology of the entropy map, shown in Fig. 10. From Fig. 11, we also note that the western radio filament pointing South-West

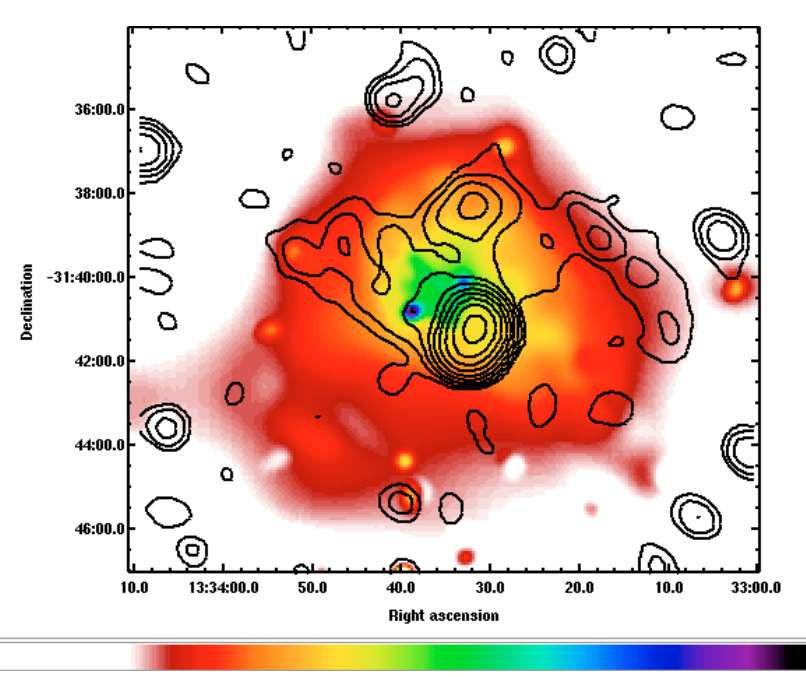

Fig. 11. VLA $1400 \mathrm{MHz}$ image of the radio halo, overlaid on the pressure map of A3562. Radio levels are $0.00015 \times(-1,1,2,4,8,16,32$, $64,128,256,512,1024) \mathrm{Jy} \mathrm{b}^{-1}$ and the resolution is $41.9^{\prime \prime} \times 35.1^{\prime \prime}$.

remarkably follows (at least in projection) the region of enhanced gas pressure commented in F04.

\subsection{Point-to-point comparison}

We performed a point-to-point analysis of the radio (flux density at $1.4 \mathrm{GHz}$ and $332-1400 \mathrm{MHz}$ spectral index) and the X-ray (surface brightness, temperature, entropy, pressure) quantities, using both Chandra and XMM-Newton data. Following Govoni et al. (2001b) we constructed a grid covering the cluster region and determined the mean radio and $\mathrm{X}$-ray quantities for every grid cell, as well as the root-meansquare (rms), which can be assumed as an estimate of the error. The whole analysis has been carried out using the IDL and the 


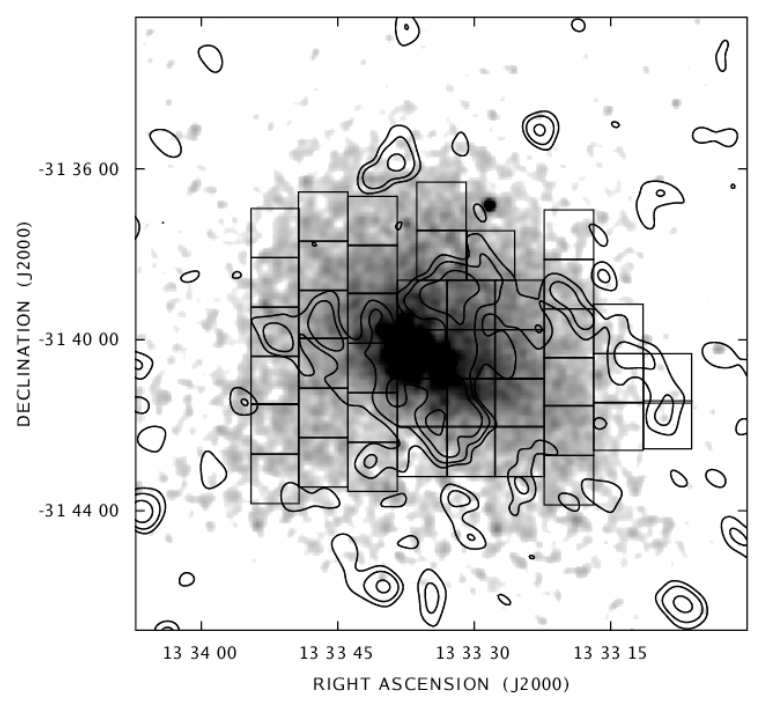

Fig. 12. Grid used for the comparison of the radio and X-ray images of the cluster A3562. The size of each grid cell is $70^{\prime \prime} \times 70^{\prime \prime}$. The grid is overlaid on the Chandra X-ray surface brightness image. Contours represent the VLA-1400 MHz image of the halo, after subtraction of the central head-tail radio galaxy J1333-3141. Radio levels are $0.00015 \times(-1,1,2,4,8,16,32,64,128,256,512,1024) \mathrm{Jy} \mathrm{b}^{-1}$. The angular resolution of the radio map is $41.9^{\prime \prime} \times 35.1^{\prime \prime}$. The X-ray image has been obtained from the Chandra raw image (resolution $2^{\prime \prime} \times 2^{\prime \prime}$ ) after a smoothing with a box of $60^{\prime \prime}$ width.

Synage ++ packages. In order to perform a proper comparison, we used an image of the halo after subtraction of the central head-tail radio galaxy J1333-3141. In Fig. 12 we show the radio halo $1.4 \mathrm{GHz}$ emission (after subtraction of the head-tail source) as contours, superposed on the Chandra X-ray surface brightness image. The figure also reports the grid used for the analysis, which covers the whole region of X-ray emission. The size of each grid cell is $70^{\prime \prime} \times 70^{\prime \prime}$, in order to select statistically independent regions.

As noted in the comparison between the radio halo and the XMM-Newton images (Sect. 6.1), we find a strong spatial correlation between the radio halo emission and the inner region of the emission from the X-ray plasma.

We investigated the presence of correlation between radio and X-ray quantities performing a Spearman test, which evaluates a rank correlation coefficient, $r_{\mathrm{s}}$, on given data arrays. The absolute value of $r_{\mathrm{s}}$ can range between 0 (no correlation is present) and 1 (perfect correlation). The test returns also $P$, the two-sided significance of the agreement of the rank correlation coefficient, $r_{\mathrm{s}}$, with the null-hypothesis of no-correlation between the data arrays. A small value of $P$ indicates a significant (anti-)correlation if $r_{\mathrm{s}}$ is (negative) positive.

The most significant correlation is given by the Chandra $\mathrm{X}$-ray surface brightness versus the radio flux density at $1400 \mathrm{MHz}$ (see Fig. 13). In the figure filled triangles represent the data from the cells with radio emission; filled dots represent the data from the cells which cover the western filament. For completeness we show also some upper limits (not included in the statistical analysis), which represent cells with no detected radio emission above the $1 \sigma$ level.

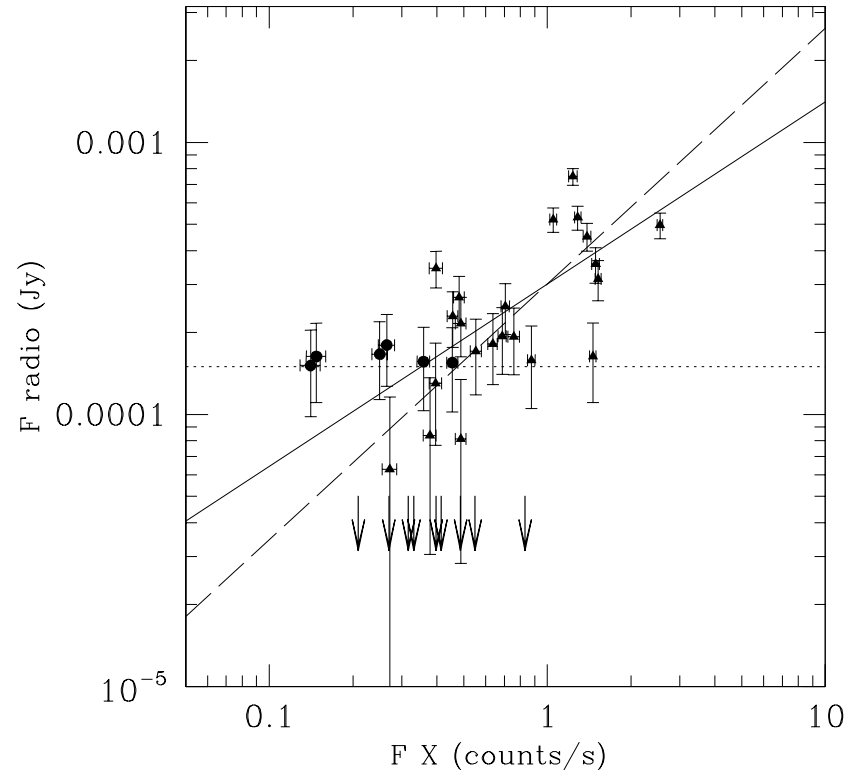

Fig. 13. Relation between the radio flux density at $1.4 \mathrm{GHz}$ and the Chandra X-ray flux for the cluster A3562. Data points represent the mean flux density in each cell of the grid constructed over the cluster region (Fig. 12). The error-bars are the rms of flux distribution. The filled dots indicate the values derived for the grid cells containing the weak filamentary structure of the radio halo extending toward SouthWest. The upper limits represent cells with no detected radio emission above the $1 \sigma$ level. The dotted line is the radio $3 \sigma$ level. The solid line is the best fit of the data including all the boxes. The long-dashed line is the best fit obtained excluding the western filament.

For these two quantities we found $r_{\mathrm{s}}=+0.65$; the probability that the relative distribution of values is not correlated is $P=1.5 \times 10^{-4}$. In this case the weighted best fit is given by $\log F_{\text {radio }}=(0.67 \pm 0.12) \log F_{\mathrm{X}}-(3.52 \pm 0.05)$, which is consistent with a sub-linear correlation, as found in some other cases (Govoni et al. 2001b). A correlation between radio flux and X-ray brightness is also confirmed by the analysis of the XMM-Newton data. It is worth noticing that if we exclude the western filament of the halo from the statistical analysis, the significance of the correlation slightly decreases to $r_{\mathrm{s}}=+0.60$ $\left(P=3.3 \times 10^{-3}\right)$, meaning that the filament plays a marginal role in the overall significance of the $F_{\text {radio }}-F_{X}$ correlation, while the slope of the correlation steepens from sub-linear to linear $\left(\log F_{\text {radio }}=(0.94 \pm 0.13) \log F_{\mathrm{X}}-(3.52 \pm 0.05)\right)$, as clear from Fig. 13.

Moreover, higher radio fluxes are measured in regions with lower values of X-ray gas temperatures and entropy levels $\left(r_{\mathrm{s}} \sim-0.6\right)$ and higher gas pressure estimates $\left(r_{\mathrm{s}} \sim+0.6\right)$. We detect weak evidence $\left(\left|r_{\mathrm{s}}\right| \leq 0.4\right)$ for a spatial correlation between the spectral index image of the radio halo (Fig. 5) and the projected X-ray quantities. We note that these results do not depend on the choice of the position of the boxes to cover the cluster emission (see Fig. 12), providing different configurations very similar results. 


\section{Cluster merger and origin of the diffuse radio emission}

Given the high quality of the X-ray and radio data and the evidence for diffuse particle acceleration from the integrated radio spectrum (Sect. 5.1), in this Section we attempt to explain the origin of the diffuse radio emission as due to electron acceleration driven by the turbulence generated in the ICM during the merger between A3562 and SC 1329-313.

The efficiency of the turbulence cascading and of the vwave-particle coupling depends on many physical quantities, which are basically unknown (e.g. the magnetic field strength and topology, the spectrum of the MHD turbulence). However, it has been recently pointed out that, independent of the details of the turbulence injection process, MS waves with scales $<100 \mathrm{kpc}$ may efficiently accelerate fast electrons in the ICM up to the energies $\left(\gamma \sim 10^{4}\right)$ required to emit the synchrotron radiation observed in the radio band (Cassano \& Brunetti 2005). The decay time scale of the MS waves is approximately $\tau_{k k} \sim$ $v_{\mathrm{MS}} / k v_{k}^{2}$ (e.g., Yan \& Lazarian 2004), where $v_{\mathrm{MS}}$ is the magnetosonic velocity, $v_{k}^{2}$ is the velocity of the turbulent eddies and $k$ is the wavenumber. Given the physical conditions in the ICM of A3562, it is:

$\tau_{k k}(\mathrm{Gyr}) \simeq 0.7 \frac{L}{\mathrm{Mpc}} \eta_{\mathrm{MS}}^{-1}$

where $\eta_{\mathrm{MS}}=E_{\mathrm{MS}} / E_{\mathrm{th}}$ is the ratio between the energy in the form of MS waves and the thermal energy in the emitting region $\left(\eta_{t} \sim 0.2-0.9\right.$ in Cassano \& Brunetti 2005 is a few times $\eta_{\mathrm{Ms}}$ here), and $L$ is the maximum injection scale of the MHD turbulence. This is comparable to the crossing time of massive subclumps through the volume of the main cluster and thus it may allow an effective spatial diffusion of the turbulence over Mpc scales with a fairly uniform intensity before being dissipated.

The proposed scenario for the origin of the radio halo in A3562 is based on the following points.

(1) Merger Kinematics - Making use of several arguments, F04 derived the kinematics of the collision between SC 1329-313 and A3562. They suggested that about 1 Gyr ago SC 1329-313 passed 500 kpc North of the core of A3562 (corresponding to $\sim 0.25$ virial radii) coming from the East, with a relative velocity of the order of $1700 \mathrm{~km} \mathrm{~s}^{-1}$, and it was gravitationally deflected toward the South-Western direction. Note that the mass ratio between A3562 and SC 1329-313 is 3:1 (Ettori et al. 1997). As a consequence of this passage, the core of A3562 acquired a velocity component in the NorthSouth direction and it started sloshing, with an oscillation amplitude of the order of $200 \mathrm{kpc}$ and a period of $\sim 1 \mathrm{Gyr}$.

(2) Magnetic field and Merger - The change of the core velocity at the oscillation apogee causes the escape of lowentropy gas. This produces the entropy tails, as discussed in F04 (see also Fig. 10 in Sect. 6.1). Since the magnetic field is frozen into the thermal plasma, we expect that the escape of the gas should flatten the profile of the field strength toward the region North of the A3562 core.

\footnotetext{
${ }^{2}$ We used $v_{k}^{2} \sim \eta_{\mathrm{MS}} \frac{E_{\mathrm{th}}}{2 \rho}, \rho=$ density of the thermal ICM, using a Kraichnan spectrum.
}

(3) Turbulence and particle acceleration - Another expected by-product of the interaction between SC 1329-313 and A3562 is the injection of turbulence in the ICM. Although the injection mechanism of the turbulence is very complex and depends on many unknown parameters, we expect that large scale turbulence may be efficiently injected in the volume swept by the head of SC 1329-313 (i.e. by the self bounded subclump which is gradually reduced during the crossing of the main cluster by the effect of the ram pressure stripping) and in the central region of A3562 by the North-South oscillation of the core. As shown by analytical arguments (Cassano \& Brunetti 2005) and by numerical simulations (Ricker \& Sarazin 2001), once injected these large scale eddies diffuse through a larger volume so that one may reasonably assume that turbulence fills at some level the region of the core of A3562 and a large fraction of the volume between the cores of the two subclusters (actually from the bridge toward the north).

The effect of the turbulence on the process of particle acceleration and amplification of the magnetic field depends on the strength of the turbulence in this region. Provided that a fraction $\sim 0.005-0.1 \times\left(10^{-3} / n_{\mathrm{th}}\right)\left(10^{8} / T\right)$ of the thermal energy of the ICM is channelled into MHD waves (depending on the specific MHD mode, see Brunetti et al. 2004, and Cassano \& Brunetti 2005), this may switch on the diffuse radio emission observed near the centre of A3562.

\subsection{Self-consistency of re-acceleration}

If electrons are re-accelerated, as indeed suggested by the curved radio spectrum of A3562 (Fig. 8), similar to Coma-C (Thierbach et al. 2003), then the source age can be estimated by the cascading time-scale of the turbulence injected on the scales of the instabilities generated during the merging process. An analysis of the entropy map limits the scale of the Rayleigh-Taylor instabilities, driven by the core oscillation, to $\sim 500 \mathrm{kpc}$; the corresponding cascading time-scale (Eq. (1)) is $\tau_{k k}=0.7\left(\frac{\eta_{\mathrm{MS}}}{0.5}\right)^{-1} \mathrm{Gyr}$; this should be considered a lower limit, since the oscillation of the core (and hence the growth of the instabilities) is still on-going.

At the same time, large scale turbulence may be injected at the passage of SC 1329-313 North of the A3562 core. In this case, the initial scale of the instabilities should be of the order of the diameter of the core of SC 1329-313 and thus the cascading time should be $\sim 1$ Gyr. We note that both these cascading time-scales are of the order of the time-scale of the merging, and of the core oscillation in A3562 (F04).

Thus the proposed scenario is self-consistent: there is enough time for the development of a turbulence cascade, necessary for particle acceleration; at the same time, the epoch of turbulence injection is recent enough to expect that turbulence is not completely dissipated at present.

\subsection{Observational evidence}

The observed correlations between radio and X-ray quantities (Sect. 6) support the proposed scenario. 
If turbulence is injected by the passage of SC 1329-313 through the cluster volume and by the oscillation of the A3562 core, then it should be fairly uniform on several hundreds kpc. Thus, if the energy density of the turbulence is smaller than that of the thermal plasma, its intensity should not significantly depend on the X-ray quantities (e.g. temperature and thermal density). In this case, once the relevant wave-damping processes are taken into account (e.g., Cassano \& Brunetti 2005), the efficiency of the particle acceleration due to MS waves should scale with the temperature as $1 / \sqrt{T}$ and thus an anticorrelation between the non-thermal emission and the higher temperature patches is expected.

On the other hand, since the magnetic field, $B$, is frozen into the ICM, higher synchrotron emissivity $\left(\propto B^{2}\right)$ is expected in regions with higher density, $n_{\text {th }}$, provided that fast electrons are accelerated in these regions. The significant correlation between radio and X-ray $\left(\propto n_{\text {th }}^{2}\right)$ flux could thus be driven by this effect. The freezing of the magnetic field into the ICM could also account for the observed anti-correlation between the radio flux and the entropy, provided that higher values of $B$ are found in regions with lower entropy. Similarly, the observed correlation between the radio flux and the thermal pressure is expected if the measured values of the pressure are mainly determined by the values of the gas density.

\subsection{The extent of the radio emission}

The observed extent of the radio emission is slightly smaller than the entropy tails of A3562. Cassano \& Brunetti (2005) calculated that cluster mergers may efficiently power giant (i.e., $\geq$ Mpc sized) radio halos only in the case of massive clusters (with $M_{200} \geq 10^{15} M_{\odot}$ ). This threshold is essentially due to the fact that the energy density (within $1 \mathrm{Mpc}^{3}$ ) of the injected turbulence in clusters with masses below this value is too small to drive efficient particle acceleration on large $(\mathrm{Mpc})$ regions, and thus the cut-off in the synchrotron spectrum falls below the typical radio-observing frequencies. A3562 is slightly below this mass threshold $\left(M_{200} \simeq 5.6 \times 10^{14} M_{\odot}\right)$, and the presence of a giant radio halo is indeed not expected by these calculations. In this case, however, it might be thought that diffuse synchrotron emission may be revealed on smaller scales in the cluster-core region and toward the North of the core, where the strength of the magnetic field is likely to be stronger (and amplified by the interaction between the two subclusters).

The clear steepening of the radio spectrum (Fig. 8) strenghtens this interpretation, since it proves that the energy of the electrons accelerated in the core region is just sufficient to emit synchrotron radiation up to $\leq \mathrm{GHz}$ frequency.

Given the shape of the observed radio spectrum, we point out that even assuming that the acceleration efficiency is maintained outside the radio emitting region, a drop in the magnetic field strength by a factor of $\sim 2$ outside this region would cause a sharp decrement of the synchrotron emissivity at $610 \mathrm{MHz}$, i.e. a factor of $\sim 7-8$.

\subsection{A radio bridge between the radio halo and J1332-3146a?}

Figure 4 and the $1.4 \mathrm{GHz}$ images presented in Fig. 9 and in G04 show outstanding features of extended radio emission. In particular, the western filament of the radio halo, the extent of J1332-3146a in the direction of the A3562 centre, and the presence of positive residuals of radio emission at $1.4 \mathrm{GHz}$ and $332 \mathrm{MHz}$ are all perfectly aligned. Furthermore, the radio/XMM-Newton overlay of Fig. 9 shows that the X-ray emission is also aligned in the same direction.

The scenario proposed in this section could account for these observed signatures, at least at a qualitative level. It seems reasonable to expect that the motion of SC 1329-313 toward South-West and the gravitational interaction with A3562 have actually produced a "channel" of turbulence, which may have affected the ICM and relativistic particles over the whole distance between the group and the cluster. If this is the case, the energy rate injected into MHD modes along the bridge should be roughly of the order of $\gtrsim 5 \times 10^{-29} \mathrm{erg} \mathrm{s}^{-1} \mathrm{~cm}^{-3}$ (here, for simplicity, we assume MS waves and a reference value of $B \sim 0.5 \mu \mathrm{G}$ and $T \sim 5 \times 10^{7} \mathrm{~K}$ ), which implies that the fluid turbulence in this low density region should be strong, from slightly subsonic to mildly supersonic depending on the efficiency of the radiation process of MS waves.

The radio images suggest that the western filament and the extent of J1332-3146a are the "peaks" of a bridge of very low brightness extended radio emission, which falls below the sensitivity limits of the current radio interferometers. This was noticed also in G04, based on $1.4 \mathrm{GHz}$ data. The new GMRT data presented here, in particular the $332 \mathrm{MHz}$ image, further strengthen this possibility. We note that the spectrum of $\mathrm{J} 1332-3146 \mathrm{a}$ is steep, with $\alpha_{240 \mathrm{MHz}}^{1.4 \mathrm{GHz}}=1.2$, which is consistent with what is found in cluster-type radio sources such as halos and relics.

\section{Summary and conclusions}

In this paper we presented and discussed new low-frequency GMRT images at $240 \mathrm{MHz}, 332 \mathrm{MHz}$ and $610 \mathrm{MHz}$ of the recently discovered radio halo at the centre of the A3562 cluster.

In the following we summarize the most important results of these observations, combined with our previous $1.4 \mathrm{GHz}$ VLA data (published in V03), and the Chandra and XMMNewton X-ray observations carried out by Ettori et al. (in prep.) and F04.

1) A3562 is characterized by extended radio emission on the cluster scale. Beyond the presence of a radio halo at its centre, the radio galaxy J1332-3146a (located South-West of A3562) shows low brightness extended emission in the direction of the radio halo, suggestive of a possible connection between the two features. This possibility is reinforced by the presence of positive residuals between these two features, both at $332 \mathrm{MHz}$ and at $1.4 \mathrm{GHz}(\mathrm{G} 04)$.

2) The radio halo in $A 3562$ is among the smallest found thus far. Its LLS is of the order of $\sim 500 \mathrm{kpc}$ going from $1.4 \mathrm{GHz}$ to $332 \mathrm{MHz}$. 
3) The point-to-point spectral index image of the halo shows a complex structure. Beyond the existence of a region with an average value of $\alpha_{332 \mathrm{MHz}}^{1.4 \mathrm{GHz}} \sim 1.5 \pm 0.1$, there are a number of knots steepening up to $\sim 2.0 \pm 0.2$. Our images also show that the spectrum of the electron populations of the radio halo and of the tailed source J1333-3141 embedded in it are clearly different.

4) With all the data available we could derive the integrated radio spectrum of the halo in the range $240 \mathrm{MHz}-1.4 \mathrm{GHz}$, with 5 data points. The spectrum is very steep between $1400 \mathrm{MHz}$ and $843 \mathrm{MHz}$, with a spectral index $\alpha_{843}^{1400 \mathrm{MHz}}$ in the range $\sim 1.9-2.3$ (V03). Between $843 \mathrm{MHz}$ and $332 \mathrm{MHz}$ the spectral index is $\alpha_{332}^{843 \mathrm{MHz}} \sim 1.3 \pm 0.2$, and below $332 \mathrm{MHz}$ the spectrum flattens to $\alpha_{240}^{332} \sim 0.4 \pm 0.7$. We also derived the spectrum for the extended source J1332$3146 \mathrm{a}$, and found that it is also steep, with $\alpha_{240 \mathrm{MHz}}^{1.4 \mathrm{GHz}}=$ $1.2 \pm 0.1$.

5) The radio and $\mathrm{X}$-ray images show that $\mathrm{A} 3562$ and the group SC 1329-313 "feel each other", i.e. the extended features of the two regions point toward each other in both bands.

6) A spatial correlation is found between the radio halo and the quantities derived from the XMM-Newton and Chandra observations, like the X-ray surface brightness, the temperature, the pseudo-entropy and pseudo-pressure maps. All the observables show an extension toward North, and a sharp edge South of the core region in A3562. A significant point-to-point linear positive correlation holds between the $1400 \mathrm{MHz}$ flux density of the halo and the X-ray flux density. Such correlation is expected, given the correlations observed between the halo emission and the pseudopressure and -entropy maps of A3562. Furthermore, the western filament of the radio halo, pointing to South-West, follows remarkably (at least in projection) a region of enhanced gas pressure.

Our results give further observational support to a scenario of cluster merger between A3562 and SC 1329-313. In particular, we discussed the hypothesis that the radio halo in A3562 is the result of particle re-acceleration induced by turbulence injected in the ICM by the passage of SC 1329-313 North of the core of A3562, and by the oscillation of the cluster core in the North-South direction. The proposed scenario may explain the properties of the radio halo, including its relatively small size, the morphology and extent of the pseudo-entropy tails as derived from the XMM-Newton observations, as well as the statistical correlations between the radio and X-ray observables.

We further speculate that the alignment of the western filament of the radio halo with the extended emission around J1332-3146a and the positive residuals of radio emission between the centre of A3562 and the group SC 1329-313, are also connected to the turbulence induced by this merger event.

It has been shown that cluster mergers involving massive $\left(M>10^{15} M_{\odot}\right)$ and luminous $\left(L_{\mathrm{X}}>10^{45} \mathrm{erg} \mathrm{s}^{-1}\right)$ clusters may develop the energy and turbulence required to produce $\mathrm{Mpc}-$ scale radio emission in the form of giant radio halos (Cassano \& Brunetti 2005). On the other hand, it might be expected that slightly less massive systems might also produce diffuse radio emission, though less extended and less powerful than giant radio halos. Indeed, the case of A3562 shows that merger events involving clusters with mass $M \sim 6 \times 10^{14} M_{\odot}$ might produce signatures in the radio band, at the detection limit of the intrumentation currently available. Therefore we expect that future observational facilities, such as LOFAR and SKA, will substantially contribute to our knowledge and understanding of the cluster merger phenomenon.

Acknowledgements. We thank the staff of the GMRT who have made these observations possible. GMRT is run by the National Centre for Radio Astrophysics of the Tata Institute of Fundamental Research. The paper is also based on observations obtained with XMM-Newton, an ESA science mission with instruments and contributions directly funded by ESA Member States and the USA (NASA). We thank R.Cassano for useful discussions, and the anonymous referee for very fruitful comments. G.B. acknowledges partial support from INAF from grant D4/03/15 and from MIUR from grant PRIN2004. A.F. acknowledges support from BMBF/DLR under grant 50 OR 0207 and MPG. The present research was carried out with partial support of the contract ASI-I-R-063-02.

\section{References}

Bacchi, M., Feretti, L., Giovannini, G., \& Govoni, F. 2003, A\&A, 400, 465

Bardelli, S., Zucca, E., Malizia, A., et al. 1996, A\&A, 305, 435

Bardelli, S., Pisani, A., Ramella, M., Zucca,, E., \& Zamorani, G. 1998a, MNRAS, 300, 589

Bardelli, S., Zucca, E., Zamorani, G., Vettolani, G., \& Scaramella, R. 1998b, MNRAS, 296, 599

Bardelli, S., Zucca, E., Zamorani, G., Moscardini, L., \& Scaramella, R. 2000, MNRAS, 312, 540

Bardelli, S., De Grandi, S., Ettori, S., et al. 2002, A\&A, 382, 17

Blasi, P., \& Colafrancesco, S. 1999, APh, 12, 169

Briel, U. G., Finoguenov, A., \& Henry, J. P. 2003, A\&A, submitted

Brunetti, G. 2003, in Matter and Energy in Clusters of Galaxies, ASP Conf. Ser., 301, 349

Brunetti G. 2004, in Outskirts of galaxy clusters: intense life in the suburbs, IAU Coll., 195, 148

Brunetti, G., Setti, G., Feretti, L., \& Giovannini, G. 2001a, MNRAS, 320,365

Brunetti, G., Setti, G., Feretti, L., \& Giovannini, G. 2001b, New Astron., 6, 1

Brunetti, G., Blasi, P., Cassano, R., \& Gabici, S. 2004, MNRAS, 350, 1174

Buote, D. A. 2001, ApJ, 553, L15

Cassano, R., \& Brunetti, G. 2005, MNRAS, 357, 1313

Colafrancesco, S. 1999, in Diffuse Thermal and Relativistic Plasma in Galaxy Clusters, ed. H. Böhringer, L. Feretti, \& P. Schuecker, MPE Report 271, 269

Churazov, E., Forman, W., Jones, C., \& Böhringer, H. 2003, ApJ, 590, 225

Dennison, B. 1980, ApJ, 239, 93

Dolag, K., Bartelmann, M., \& Lesch, H. 2002, A\&A, 387, 383

Eilek, J. A. 1979, ApJ, 230, 373

Ettori, S., Fabian, A. C., \& White, D. A. 1997, MNRAS, 289, 787

Ettori, S., Bardelli, S., De Grandi, S., et al. 2000, MNRAS, 318, 239 
Feretti, L. 2003, ASP Conf. Ser., 310, 143

Feretti, L., Orrù, E., Brunetti, G., Giovannini, G., Kassim, N., \& Setti, G. 2004, A\&A, 423, 111

Finoguenov, A., Henriksen, M. J., Briel, U. G., de Plaa, J., \& Kaastra, J. S. 2004, ApJ, 611, 811 (F04)

Fujita, Y., Takizawa, M., \& Sarazin, C. L. 2003, ApJ, 584, 190

Giacintucci, S., Venturi, T., Bardelli, S., Dallacasa, D., \& Zucca, E. 2004, A\&A, 419, 71 (G04)

Govoni, F., Feretti, L., Giovannini, G., et al. 2001a, A\&A, 376, 803

Govoni, F., Enßlin, T. A., Feretti, L., et al. 2001b, A\&A, 369, 441

Govoni, F., Markevitch, M., Vikhlinin, A., et al. 2004, ApJ, 605, 695

Liang, H., Hunstead, R. W., Birkinshaw, M., \& Andreani, P. 2000, ApJ, 544, 686

Murgia M. 2001, Ph.D. Thesis, University of Bologna

Parma, P., Murgia, M., de Ruiter, H. R., \& Fanti, R. 2002, NewAR, 46, 313

Petrosian, V. 2001, ApJ, 557, 560

Ricker, P. M., \& Sarazin, C. L. 2001, ApJ, 561, 621
Roettiger, K., Loken, C., \& Burns, J. O. 1997, ApJ, 109, 307

Sarazin, C. L. 2004, in X-ray and radio connections meeting, ed. L. O. Sjouwerman, \& K. K. Dyer [arXiv:astro-ph/0406181]

Schlikeiser, R., Sievers, A., \& Thiemann, H. 1987, A\&A, 182, 21

Schuecker, P., Bhringer, H., Reiprich, T. H., \& Feretti, L. 2001, A\&A, 378, 408

Thierbach, M., Klein, U., \& Wielebinski, R. 2003, A\&A, 397, 53

Venturi, T., Bardelli, S., Morganti, R., \& Hunstead, R. W. 1997, MNRAS, 285, 898

Venturi, T., Bardelli, S., Morganti, R., \& Hunstead, R. W. 1998, MNRAS, 298, 1113

Venturi, T., Morganti, R., Bardelli, S., Dallacasa, D., \& Hunstead, R. W. 1999, ASPC, 176, 256

Venturi, T., Bardelli, S., Morganti, R., \& Hunstead, R. W. 2000, MNRAS, 314, 594 (V00)

Venturi, T., Bardelli, S., Dallacasa, D., et al. 2003, A\&A, 402, 913 (V03)

Yan, H., \& Lazarian, A. 2004, ApJ, 614, 757 